\title{
Papers
}

\section{Efficacy of vitamin B-6 in the treatment of premenstrual syndrome: systematic review}

\author{
Katrina M Wyatt, Paul W Dimmock, Peter W Jones, P M Shaughn O’Brien
}

\begin{abstract}
Objective To evaluate the efficacy of vitamin B-6 in the treatment of premenstrual syndrome.

Design Systematic review of published and unpublished randomised placebo controlled trials of the effectiveness of vitamin B-6 in the management of premenstrual syndrome.

Subjects Nine published trials representing 940 patients with premenstrual syndrome.

Main outcome measures Proportion of women whose overall premenstrual symptoms showed an improvement over placebo. A secondary analysis was performed on the proportion of women whose premenstrual depressive symptoms showed an improvement over placebo.

Results Odds ratio relative to placebo for an improvement in overall premenstrual symptoms was 2.32 (95\% confidence interval 1.95 to 2.54$)$. Odds ratio relative to placebo for an improvement in depressive symptoms was 1.69 (1.39 to 2.06) from four trials representing 541 patients.

Conclusion Conclusions are limited by the low quality of most of the trials included. Results suggest that doses of vitamin B-6 up to $100 \mathrm{mg}$ /day are likely to be of benefit in treating premenstrual symptoms and premenstrual depression.
\end{abstract}

\section{Introduction}

The UK Department of Health and the Medical Control Agency have recently published recommendations to restrict the dose of vitamin B-6 available generally to $10 \mathrm{mg}$ and to limit the dose sold by a pharmacist to less than $50 \mathrm{mg}$. ${ }^{1}$ Vitamin B-6 is often used to treat premenstrual syndrome without clear evidence of its efficacy, hence it is timely to re-evaluate vitamin B-6 in the treatment of premenstrual syndrome.

Premenstrual syndrome exists when women complain of regularly recurring psychological or somatic symptoms, or both, which occur specifically during the luteal phase of the menstrual cycle and which are relieved by the onset of, or during, menstruation. Symptoms can be severe enough to disrupt every day life. ${ }^{2}$ Mild physiological symptoms occur in approximately $95 \%$ of all women of reproductive age. Approximately $5 \%$ of symptomatic women complain of such severe symptoms that their lives are completely disrupted. ${ }^{3}$

Somatic symptoms of premenstrual syndrome include bloating, weight gain, mastalgia, abdominal discomfort and pain, lack of energy, headache, and exacerbations of chronic illnesses such as asthma, allergies, epilepsy, or migraine. Commonly reported affective changes are dysphoria, irritability, anxiety, tension, aggression, feelings of being unable to cope, and a sense of loss of control. ${ }^{4}$

Since the original description of the syndrome in $1931^{5}$ numerous hypotheses have been advanced to explain premenstrual syndrome, but to date the pathogenesis remains unclear and speculative. ${ }^{6}$ This uncertainty reflects the many treatments available ${ }^{7}$; one reviewer suggested that there were as many as 327 different treatments for premenstrual syndrome. ${ }^{6}$ Most interventions, however, have been on the basis of informal observations, retrospective data collection, or inadequately controlled trials.

The recommended dietary allowance for vitamin B-6 is around $2.0 \mathrm{mg} /$ day, depending on age and protein intake, ${ }^{8}$ and deficiency of vitamin B-6 is rare. ${ }^{9}$ Excessive ingestion (2000-6000 mg) of vitamin B-6 causes peripheral neuropathy, ${ }^{10-17}$ and doses of 200 $\mathrm{mg}$ /day may cause similar, although probably reversible, effects. ${ }^{18}$

Because the efficacy of vitamin B-6 has not yet been proved, and in light of recent government recommendations, we undertook a systematic review of published and unpublished randomised controlled trials where efficacy of vitamin B-6 was compared with placebo in women with premenstrual syndrome.

\section{Methods}

\section{Trials}

We found reports of published and unpublished clinical trials by searching medical databases for trials of vitamin B-6 (pyridoxine) in the management of premenstrual syndrome (MeSH terms used were premenstrual syndrome and pyridoxine, together with title and abstract searches for keywords vitamin and pyridoxine, premenstrual syndrome, PMT, LLPDD, and PMDD). We also contacted relevant pharmaceutical companies manufacturing vitamin B-6 preparations. The trials were identified by searching Embase (1988 to 1996), Medline (1966 to 1998), Psychlit (1974 to 1997), Cinahl (1982 to 1997), and the database of

\section{Academic \\ Department of Obstetrics and Gynaecology, North Staffordshire Hospital, Stoke on Trent ST4 6QG Katrina M Wyatt, non-clinical lecturer in reproductive endocrinology Paul W Dimmock, researcher P M Shaughn O'Brien, professor \\ Department of Mathematics, Keele University, Keele ST5 5BG \\ Peter W Jones, professor \\ Correspondence to: Dr Wyatt mea10@keele.ac.uk}

BMJ 1999;318:1375-81 
the Cochrane Controlled Trials Register. We searched references cited in all included and excluded trials to identify any missing studies. All languages were included. Trials investigating the effect of vitamin B-6 on premenstrual symptoms were included if they were randomised, placebo controlled, double blind, parallel, or crossover studies (where the first treatment period could be treated as a parallel trial) for which data could be acquired. We also included studies investigating the effect of multivitamin supplements on premenstrual symptoms, when the vitamin preparation contained 50 $\mathrm{mg}$ or more of vitamin B-6.

Data extraction and outcome measures

We extracted the data from each trial that met the inclusion criteria. When there were insufficient data presented for inclusion, we contacted authors for further details. Data on the dosage and preparation of vitamin B-6 were collected. The main outcome measure was an improvement in overall premenstrual symptoms. Combined or overall symptoms was chosen in an attempt to overcome the clinical heterogeneity concerned with the measurement and scoring of premenstrual symptoms. As a secondary outcome we recorded the improvement in depressive premenstrual symptoms when suitable information was presented. Where possible we also recorded the number of women withdrawing from treatment and those complaining of side effects.

\section{Quality assessment}

We assessed the quality of each trial with two different methods. Firstly, we assessed the methodology of each trial with a scale developed by Jadad and colleagues. ${ }^{19}$ This scale assesses the randomisation and double blinding and reports of dropouts and withdrawals. Secondly, we developed a second quality scale to assess the trials for study design, reproducibility, and statistical analysis. The eight point scale measured: preliminary diagnosis of premenstrual syndrome for all participants in the trial; confirmation that no other vitamin supplements or oral contraceptives were being concurrently taken; the randomisation procedure described in detail; a power calculation to justify participant numbers, or $>65$ participants in each arm of the study; a single clearly stated dose of vitamin B-6 only; reproducible measurement of premenstrual symptoms such as use of visual analogue scales or Moos' menstrual distress questionnaire; clear presentation of results; and a description of the number and reason for trial withdrawals. One point was awarded for each category. Each trial was independently scored by KMW and PWD, and any areas of disagreement arbitrated by PMSO. A predetermined criterion for the recognition of the highest quality trials was established. A score of 3 or more was required in the Jadad score (as recommended by Jadad and colleagues ${ }^{19}$ ) and a score of 6 or more was required in our eight item quality assessment.

\section{Statistical analysis}

Where dichotomous data were presented, odds ratios with $95 \%$ confidence intervals were generated for the primary and, where possible, secondary outcome in each trial. ${ }^{20}$ We calculated odds ratios by categorising patients with a subjective improvement as "better," and those with no change or worse symptoms as "not better." Odds ratios of $>1$ indicated that an event was more likely to occur in the group receiving vitamin B-6 than in the group receiving placebo. We calculated an overall odds ratio with fixed and random effects models. ${ }^{20}$ Homogeneity was tested for with a $\chi^{2}$ test, with $\mathrm{P}<0.05$ indicating significant heterogeneity. For trials using continuous measures of premenstrual symptomology, we calculated a standardised mean difference (or effect size), and converted to an odds ratio using a relation given by Hasselblad and Hedges. ${ }^{21}$

To detect bias (such as publication and location) within the analysed trials, we constructed a funnel plot. ${ }^{22}$ To assess quantitatively the asymmetry of the funnel plot we used the method of Egger and colleagues. ${ }^{22}$ Briefly, a linear regression of the standard normal deviate defined as the odds ratio is divided by its $\mathrm{SE}$ against precision (inverse of the standard error). A regression line which passes through the origin of the plot (within error limits) indicates symmetry and hence the absence of bias.

\section{Results}

We identified 25 published trials. ${ }^{2}{ }^{12}{ }^{23-45}$ Of these, four were excluded because they did not include a placebo group, ${ }^{3438} 3943$ two were retrospective studies, ${ }^{4041}$ and nine presented quantitative data that could not be analysed, ${ }^{2} 182327$ 35-37 $4245^{45}$ leaving 10 placebo controlled trials. ${ }^{24-26}{ }^{28-44}$ Two of the trials had two separate dosing regimens and were effectively treated as four studies. Only one trial contained details of the method of randomisation..$^{25}$

\section{Quality assessment of the trials}

Three of the included trials met the Jadad scale cut off point of a score of $\geqslant 3 .^{25}{ }^{2633}$ However, only one of the trials included in the meta-analysis scored 6 on our eight point criteria for classification as a high quality trial. ${ }^{31}$ Unfortunately this trial had too few subjects to achieve sufficient power and had a low Jadad score. The overall methodology of the trials was poor, with none of the included trials justifying patient numbers with a power calculation. Three of the 10 studies reported the number of withdrawals from a trial, together with reasons, and side effects when taking either vitamin B-6 or placebo. ${ }^{26} 3033$

Of the nine trials included in the meta-analysis (one trial was excluded owing to statistical heterogeneity), a statement regarding whether women taking oral contraceptives were excluded from the trial was reported in three trials, ${ }^{2528}{ }^{31}$ three other trials do not mention this as an exclusion criterion, ${ }^{24}{ }^{29}{ }^{30}$ and one trial excluded women "on medication."33 Two trials ${ }^{26} 32$ included women who were taking the contraceptive pill, but they both flagged those women who did and analysed them separately and found no statistically significant difference between those taking oral contraceptives and those not taking oral contraceptives.

As none of the trials met both our and the Jadad quality criteria, we did not take the quality score into account when considering trials for inclusion. Tables 1 and 2 present the quality scores for included and excluded trials.

We calculated the odds ratios with both fixed and random effects models. Minimal difference was found 
Table 1 Characteristics of studies included in meta-analysis of vitamin B-6 in premenstrual syndrome

\begin{tabular}{|c|c|c|c|c|c|c|c|}
\hline Study & Participants & Intervention & Outcome measures & Reported results & $\begin{array}{l}\text { Withdrawals and } \\
\text { side effects* }\end{array}$ & $\begin{array}{c}\text { Jadad } \\
\text { (authors') } \\
\text { quality } \\
\text { score }\end{array}$ & Comments \\
\hline Colin $1982^{24}$ & $\begin{array}{l}32 \text { with diagnosed } \\
\text { mastalgia; } 17 \text { given } \\
\text { vitamin B-6, } 15 \text { given } \\
\text { placebo }\end{array}$ & $\begin{array}{l}500 \mathrm{mg} / \text { day for } \\
4 \text { months }\end{array}$ & Mastalgia symptoms worse or better & $\begin{array}{l}58 \% \text { better taking } \\
\text { vitamin B- } 6,59 \% \\
\text { better taking } \\
\text { placebo }\end{array}$ & None reported & $1(3)$ & $\begin{array}{l}\text { Only studied mastalgia } \\
\text { Exclusion of women on oral } \\
\text { contraceptives not mentioned }\end{array}$ \\
\hline Barr $1984^{25}$ & $\begin{array}{l}\text { 48; crossover study } \\
\text { design }\end{array}$ & $\begin{array}{l}100 \mathrm{mg} / \text { day } \\
\text { day } 10 \text { to day } \\
3 \text { after cycle } \\
\text { for } 2 \text { months }\end{array}$ & $\begin{array}{l}\text { Depression, irritability, tiredness, } \\
\text { headache, stomach ache, and } \\
\text { swollen breasts, abdomen, fingers, } \\
\text { or ankles }\end{array}$ & $\begin{array}{l}\text { Vitamin B-6 } \\
\text { significantly better } \\
\text { than placebo }\end{array}$ & None reported & $3(4)$ & $\begin{array}{l}\text { "Most" women were not on } \\
\text { oral contraceptives }\end{array}$ \\
\hline $\begin{array}{l}\text { Williams et al } \\
1985^{26}\end{array}$ & $\begin{array}{l}434 \text { with diagnosed } \\
\text { premenstrual syndrome; } \\
204 \text { given vitamin B-6, } \\
230 \text { given placebo }\end{array}$ & $\begin{array}{l}100 \mathrm{mg} / \text { day } \\
(55 \%) \\
200 \mathrm{mg} / \text { day } \\
(29 \%) \\
50 \mathrm{mg} / \text { day } \\
(3 \%)\end{array}$ & $\begin{array}{l}\text { Depression, irritability, tension, } \\
\text { violence, coordination, breast } \\
\text { tenderness, bloating, headache, acne } \\
\text { rated on a } 4 \text { point scale }\end{array}$ & $\begin{array}{l}\text { Significant } \\
\text { improvement of } \\
\text { global symptoms } \\
\text { only for vitamin } \\
\text { B-6 over placebo }\end{array}$ & $\begin{array}{l}\text { Owing to side } \\
\text { effects } 11 \text { women } \\
\text { withdrew on vitamin } \\
\text { B- } 6 \text { and } 8 \text { withdrew } \\
\text { on placebo }\end{array}$ & $3(4)$ & $\begin{array}{l}\text { Patients included were allowed } \\
\text { drugs: } 74 \text { psychotropics; } 60 \\
\text { analgesics; } 33 \text { diuretics; } 100 \\
\text { oral contraceptives Only } \\
\text { analgesia significantly affected } \\
\text { analysis }\end{array}$ \\
\hline $\begin{array}{l}\text { Chakmakjian et } \\
\text { al } 1985^{28}\end{array}$ & $\begin{array}{l}31 \text { with diagnosed } \\
\text { premenstrual syndrome } \\
\text { from hospital and general } \\
\text { population; crossover } \\
\text { study design }\end{array}$ & $\begin{array}{l}\text { Multivitamin } \\
\text { containing } 300 \\
\text { mg vitamin } \\
\text { B-6/day for } 2 \times \\
3 \text { cycles }\end{array}$ & $\begin{array}{l}19 \text { symptoms in } 4 \text { subgroups: } \\
\text { PMT-A, PMT-C, PMT-D, and PMT-H }\end{array}$ & $\begin{array}{l}\text { Significant effect } \\
\text { for symptom } \\
\text { groups PMT-A and } \\
\text { PMT-C but not for } \\
\text { PMT-H and PMT-D }\end{array}$ & None reported & $2(3)$ & $\begin{array}{l}\text { Women on oral contraceptives } \\
\text { excluded }\end{array}$ \\
\hline $\begin{array}{l}\text { Smallwood et al } \\
1986^{29}\end{array}$ & $\begin{array}{l}42 \text { hospital patients "with } \\
\text { severe cyclical mastalgia"; } \\
\text { crossover study design }\end{array}$ & $\begin{array}{l}200 \mathrm{mg} / \text { day for } \\
2 \times 2 \text { cycles }\end{array}$ & $\begin{array}{l}\text { Breast pain and tenderness rated on } \\
\text { chart and visual analogue scale }\end{array}$ & $\begin{array}{l}\text { No significant } \\
\text { effect }\end{array}$ & None reported & $2(4)$ & $\begin{array}{l}\text { Only studied mastalgia } \\
\text { Exclusion of women on oral } \\
\text { contraceptives not mentioned }\end{array}$ \\
\hline Stewart $1987^{30 *}$ & $\begin{array}{l}222 \text { " with premenstrual } \\
\text { syndrome } \\
\text { symptomology"; } 49 \text { given } \\
200 \text { mg vitamin B-6, } 70 \\
\text { given placebo } 46 \text { given } \\
100 \text { mg vitamin B-6, } 58 \\
\text { given placebo }\end{array}$ & $\begin{array}{l}\text { Multivitamin } \\
\text { containing } 100 \\
\text { mg vitamin B-6 } \\
\text { day } 1 \text { to day } \\
14 \text { then } 200 \\
\text { mg for } 4 \\
\text { cycles } \\
\text { Multivitamin } \\
\text { containing } \\
50 \text { mg vitamin } \\
\text { B-6 day } 1 \text { to } \\
\text { day } 14 \text { then } \\
100 \mathrm{mg}\end{array}$ & $\begin{array}{l}19 \text { symptoms in } 4 \text { subgroups: } \\
\text { PMT-A, PMT-C, PMT-D, and PMT-H } \\
\text { rated worse, no better, slightly } \\
\text { better, substantially better, or cured }\end{array}$ & $\begin{array}{l}100 \mathrm{mg} \text { and } 200 \\
\mathrm{mg} \text { significantly } \\
\text { better than } \\
\text { placebo, } 50 \mathrm{mg} \\
\text { and } 100 \mathrm{mg} \text { not } \\
\text { significantly better } \\
\text { than placebo }\end{array}$ & $\begin{array}{l}30 \text { withdrew on } \\
\text { placebo, } 26 \\
\text { withdrew on vitamin } \\
\text { B-6, } 5 \text { had side } \\
\text { effects on vitamin } \\
\text { B-6 and } 1 \text { on } \\
\text { placebo, } 17 \\
\text { withdrew on } \\
\text { placebo, } 22 \\
\text { withdrew on vitamin } \\
\text { B-6, } 5 \text { had side } \\
\text { effects on vitamin } \\
\text { B-6 and } 2 \text { on } \\
\text { placebo }\end{array}$ & $1(4)$ & $\begin{array}{l}\text { Exclusion of women on oral } \\
\text { contraceptives not mentioned } \\
\text { Study blinded to patient only }\end{array}$ \\
\hline $\begin{array}{r}\text { Doll et al } \\
1989^{32}\end{array}$ & $\begin{array}{l}32 \text { with moderate to } \\
\text { severe premenstrual } \\
\text { symptoms in previous } \\
\text { year recruited from } \\
\text { general practitioner; } \\
\text { crossover study design }\end{array}$ & $\begin{array}{l}50 \mathrm{mg} / \text { day for } \\
3 \text { cycles with } \\
\text { one washout } \\
\text { cycle between }\end{array}$ & $\begin{array}{l}3 \text { symptom groups: emotional, } \\
\text { somatic, and menstrual }\end{array}$ & $\begin{array}{l}\text { Significant effect } \\
\text { on emotional } \\
\text { symptoms }\end{array}$ & None reported & $2(5)$ & $\begin{array}{l}\text { Included women on oral } \\
\text { contraceptives but data } \\
\text { flagged }\end{array}$ \\
\hline $\begin{array}{l}\text { London et al } \\
\qquad 1991^{33}\end{array}$ & $\begin{array}{l}44 \text { with self perceived } \\
\text { premenstrual syndrome } \\
\text { recruited form the } \\
\text { general population; } 28 \\
\text { given vitamin B-6, } 16 \\
\text { given placebo }\end{array}$ & $\begin{array}{l}2 \text { parallel arms } \\
\text { Multivitamin } \\
\text { containing } 300 \\
\text { mg vitamin B-6 } \\
\text { or } 600 \mathrm{mg} \\
\text { vitamin } \\
\text { B-6/day for } 3 \\
\text { cycles }\end{array}$ & $\begin{array}{l}19 \text { symptoms in } 4 \text { subgroups: } \\
\text { PMT-A, PMT-C, PMT-D, and PMT-H } \\
\text { with London variation }\end{array}$ & $\begin{array}{l}\text { Significant effect } \\
\text { observed }\end{array}$ & $\begin{array}{l}\text { Withdrawals: } \\
1 \text { taking placebo, } \\
1 \text { taking } 300 \mathrm{mg} \\
\text { vitamin B-6, } \\
2 \text { taking } 600 \mathrm{mg} \\
\text { vitamin B-6, } 1 \text { noted } \\
\text { "tingling in fingers" } \\
\text { at the end of the } \\
600 \mathrm{mg} \text { study }\end{array}$ & $3(5)$ & $\begin{array}{l}\text { Exclusion of women on oral } \\
\text { contraceptives not mentioned } \\
\text { Women on "medication" } \\
\text { excluded }\end{array}$ \\
\hline
\end{tabular}

${ }^{*}$ Neurological side effects reported in 1 of 934 patients while taking $600 \mathrm{mg} /$ day vitamin B-6 as part of multivitamin preparation. ${ }^{33}$

between the two methods, and so the results from the more conservative random effects model were used. The overall odds ratio in favour of vitamin B-6 was 1.57 (95\% confidence interval 1.40 to 1.77 ). One trial caused significant heterogeneity in the overall odds ratio (homogeneity test, $\mathrm{P}=<0.001),{ }^{44}$ and removal of this trial resulted in homogeneity (homogeneity test, $\mathrm{P}=0.187$ ). The recalculated odds ratio in favour of vitamin B-6 was 2.32 (1.95 to 2.54). This heterogeneity may be due to an unexpectedly high placebo effect, which resulted in an odds ratio in favour of placebo. It should be noted, however, that the overall odds ratio remained significantly in favour of vitamin B-6 even with the inclusion of this trial. Table 1 lists the nine included trials, and table 2 lists the other 16 trials that were identified. Figure 1 shows the odds ratio and dosage for each of the nine included trials.

We extracted data on the efficacy of vitamin B-6 in treating depressive premenstrual symptoms from five trials. ${ }^{26}{ }^{28}{ }^{31-33}$ Figure 2 shows the odds ratio for each trial and the term used by those trials to describe depression. The overall odds ratio in favour of vitamin B-6 was 2.12 (1.80 to 2.48; homogeneity test $\mathrm{P}<0.001$ ). This combined result showed significant heterogeneity with both fixed and random effects models. Exclusion of one trial ${ }^{31}$ gave an overall odds ratio of 1.69 (1.39 to 2.06), which was homogeneous (homogeneity test, $\mathrm{P}=0.079)$. 
Table 2 Characteristics of studies not included in meta-analysis of vitamin B-6 in premenstrual syndrome

\begin{tabular}{|c|c|c|c|c|c|c|c|}
\hline Study & No of participants & Intervention & $\begin{array}{l}\text { Reason for } \\
\text { exclusion }\end{array}$ & Reported results & Side effects* & $\begin{array}{l}\text { Jadad (authors') } \\
\text { quality score }\end{array}$ & Comments \\
\hline Kerr $1977^{34}$ & 70 & $40-100 \mathrm{mg}$ & $\begin{array}{l}\text { Not placebo } \\
\text { controlled }\end{array}$ & Considerable benefit & None observed & $0(1)$ & \\
\hline Day $1979^{35}$ & 57 & $100 \mathrm{mg}$ & No detailed data & $\begin{array}{l}\text { Improvement in } 63 \% \\
\text { of patients on } \\
\text { pyroxidine }\end{array}$ & $\begin{array}{l}\text { No significant side effects } \\
\text { observed }\end{array}$ & $0(3)$ & \\
\hline O'Brien $1987^{2}$ & $\begin{array}{l}\text { 53; crossover study } \\
\text { design }\end{array}$ & $100 \mathrm{mg}$ & $\begin{array}{l}\text { Data not suitable for } \\
\text { meta-analysis }\end{array}$ & $\begin{array}{l}\text { No benefit of drug } \\
\text { over placebo }\end{array}$ & None reported & $1(4)$ & $\begin{array}{l}24 \text { patients preferred pyridoxine } v \\
14 \text { taking placebo, but results } \\
\text { not significant }\end{array}$ \\
\hline $\begin{array}{l}\text { Abraham and } \\
\text { Hargrove } 1980^{36}\end{array}$ & 25 & $500 \mathrm{mg}$ & $\begin{array}{l}\text { Data not suitable for } \\
\text { meta-analysis }\end{array}$ & $\begin{array}{l}21 \text { patients showed } \\
\text { significant } \\
\text { improvement over } \\
\text { placebo }\end{array}$ & None reported & $2(5)$ & \\
\hline $\begin{array}{l}\text { Mattes and Martin } \\
1982^{37}\end{array}$ & 3 & $50 \mathrm{mg}$ & $\begin{array}{l}\text { Anecdotal study, no } \\
\text { assessment of } \\
\text { symptoms }\end{array}$ & $\begin{array}{l}\text { Some beneficial } \\
\text { effects }\end{array}$ & None reported & $2(1)$ & \\
\hline $\begin{array}{l}\text { Goei and Abraham } \\
1983^{38}\end{array}$ & 31 & $\begin{array}{l}\text { Multivitamin } \\
\text { containing } \\
150-600 \mathrm{mg} \\
\text { vitamin B-6 }\end{array}$ & $\begin{array}{l}\text { Not placebo } \\
\text { controlled }\end{array}$ & $\begin{array}{l}\text { Significant decrease } \\
\text { in symptoms in all } \\
\text { patients }\end{array}$ & $\begin{array}{l}5 \text { patients reported } \\
\text { gastrointestinal side } \\
\text { effects }\end{array}$ & $1(5)$ & Multivitamin \\
\hline $\begin{array}{l}\text { Harrison et al } \\
1984^{45}\end{array}$ & 30 & $50-150 \mathrm{mg}$ & $\begin{array}{l}\text { Sequential design; } \\
\text { data not suitable for } \\
\text { analysis }\end{array}$ & No benefit & $\begin{array}{l}>100 \mathrm{mg} / 3 \mathrm{~g} \text { tryptophan } \\
\text { caused drowsiness, } \\
\text { nausea, headache, } \\
\text { overstimulation }\end{array}$ & $1(5)$ & $\begin{array}{l}\text { Cointervention each tablet: } 50 \mathrm{mg} \\
\text { vitamin } \mathrm{B}-6+1.5 \mathrm{~g} \text { of } \\
\text { tryptophan; up to } 3 \text { tablets per } \\
\text { day }\end{array}$ \\
\hline Hagen et al $1985^{27}$ & 34 & $100 \mathrm{mg}$ & $\begin{array}{l}\text { Data not suitable for } \\
\text { meta-analysis }\end{array}$ & No significant effect & $\begin{array}{l}6 \text { reported nausea: } \\
1 \text { taking vitamin B-6 and } \\
1 \text { taking placebo }\end{array}$ & $3(5)$ & $\begin{array}{l}\text { Large phase effect: first } \\
\text { treatment, whether placebo or } \\
\text { vitamin B-6, gave same result } \\
\text { Women on oral contraceptives } \\
\text { excluded }\end{array}$ \\
\hline Fuchs et al $1985^{39}$ & 16 & $\begin{array}{l}\text { Multivitamin } \\
\text { containing } \\
300-600 \mathrm{mg} \\
\text { vitamin B-6 }\end{array}$ & $\begin{array}{l}\text { Not placebo } \\
\text { controlled }\end{array}$ & Beneficial effect & None reported & $0(2)$ & $\begin{array}{l}\text { Multivitamin study centred on } \\
\text { analysis of liver enzymes }\end{array}$ \\
\hline $\begin{array}{l}\text { Malmgren et al } \\
1987^{18}\end{array}$ & 19 & $300 \mathrm{mg}$ & No data & $\begin{array}{l}\text { No significant effect } \\
\text { over placebo }\end{array}$ & None reported & $1(4)$ & $\begin{array}{l}\text { Study centred on uptake of } \\
\text { platelet serotonin }\end{array}$ \\
\hline Brush et al $1988^{40}$ & 630 & $40-200 \mathrm{mg}$ & $\begin{array}{l}\text { Retrospective study, } \\
\text { not placebo } \\
\text { controlled }\end{array}$ & $\begin{array}{l}40 \% \text { "good" } \\
\text { response } 65 \%-88 \% \\
\text { partial response } \\
\text { depending on dose }\end{array}$ & $\begin{array}{l}7 \text { patients reported } \\
\text { indigestion, } 5 \text { reported } \\
\text { nausea, } 3 \text { reported breast } \\
\text { soreness } \\
\text { No neuropathy reported }\end{array}$ & $0(1)$ & $\begin{array}{l}\text { Other side effects were termed } \\
\text { "coincidental" and included } \\
\text { worse premenstrual symptoms } \\
\text { and depression }\end{array}$ \\
\hline Brush $1988^{41}$ & 336 & $\leqslant 200 \mathrm{mg}$ & $\begin{array}{l}\text { Retrospective study, } \\
\text { not placebo } \\
\text { controlled }\end{array}$ & $\begin{array}{l}70 \% \text { reported good } \\
\text { or partial response }\end{array}$ & $\begin{array}{l}8 \text { patients reported } \\
\text { nausea, } 5 \text { dizziness, } \\
6 \text { mild tingling or } \\
\text { numbness }\end{array}$ & $0(1)$ & $\begin{array}{l}5 \text { of the patients reporting } \\
\text { tingling or numbness were on } \\
\text { the highest dose }(200 \mathrm{mg})\end{array}$ \\
\hline $\begin{array}{l}\text { Van den Berg et al } \\
1989^{42}\end{array}$ & 19 & $120 \mathrm{mg}$ & $\begin{array}{l}\text { Data not suitable for } \\
\text { meta-analysis }\end{array}$ & No beneficial effect & None reported & $1(4)$ & \\
\hline $\begin{array}{l}\text { Berman et al } \\
1990^{43}\end{array}$ & 28 & $250 \mathrm{mg}$ & $\begin{array}{l}\text { Not placebo } \\
\text { controlled }\end{array}$ & $\begin{array}{l}\text { Some favourable } \\
\text { trends in } \\
\text { improvement of } \\
\text { symptoms }\end{array}$ & None reported & $0(5)$ & \\
\hline $\begin{array}{l}\text { De Souza et al } \\
1996^{44}\end{array}$ & $\begin{array}{l}\text { 44; crossover study } \\
\text { design }\end{array}$ & $\begin{array}{l}50 \mathrm{mg} \text { every day } \\
\text { for } 2 \times 1 \text { cycle }\end{array}$ & $\begin{array}{l}\text { Statistically } \\
\text { heterogeneous }\end{array}$ & No significant effect & None reported & $2(4)$ & $\begin{array}{l}4 \text { crossovers for magnesium, } \\
\text { vitaminB-6, magnesium + vitamin } \\
\text { B-6, placebo }\end{array}$ \\
\hline
\end{tabular}

${ }^{\star}$ Of 1395 patients taking vitamin B-6, six reported neurological side effects: five patients taking $200 \mathrm{mg} / \mathrm{day}$ and one patient taking 100-200 mg/day.

Figure 3 shows a dose response plot of vitamin B-6 dosage against the odds ratio for each of the nine trials. There was no correlation between the amount of vitamin B-6 given and its efficacy.

Figure 4 details a funnel plot of the included trials. Regression analysis of this plot indicated no significant asymmetry (intercept $-0.25(90 \%$ confidence interval -0.88 to 0.38$) \mathrm{P}=0.49),{ }^{22}$ and thus no evidence of bias.

\section{Discussion}

The conclusions that can be drawn from our systematic review are limited. Although the results from the available data suggest that vitamin B-6 is more effective than placebo, there is insufficient evidence of high enough quality to give a confident recommendation for using vitamin B-6 in the treatment of premenstrual syndrome. For example, the evidence from the overall analysis would be more compelling if there were large scale rigorous trials with sufficient power to detect a clinically significant effect. To detect a medium effect size $(0.5)$ at a significance level of 0.05 and $80 \%$ power, approximately 65 subjects would be required in each arm, using a two tailed test. Only one of the nine studies included a sufficient number of patients, ${ }^{26}$ and all the other trials had low statistical power $(<70 \%)$. The methodologies of each trial varied considerably using differing dosage regimens and different outcome measures, which makes intercomparisons difficult. In addition, the possible inclusion of women taking oral contraceptives complicates the overall analysis of the effect of vitamin B-6 on premenstrual syndrome as the vitamin may be treating pill induced premenstrual symptoms or depression.

An overall odds ratio was calculated from the nine included trials. Of these trials, two ${ }^{24}$ only studied the effect of vitamin B-6 on mastalgia and three ${ }^{283033}$ used the multivitamin preparation Optivite (Optimox, Torrance, CA, USA). We believed it was appropriate to 


\section{Trials}

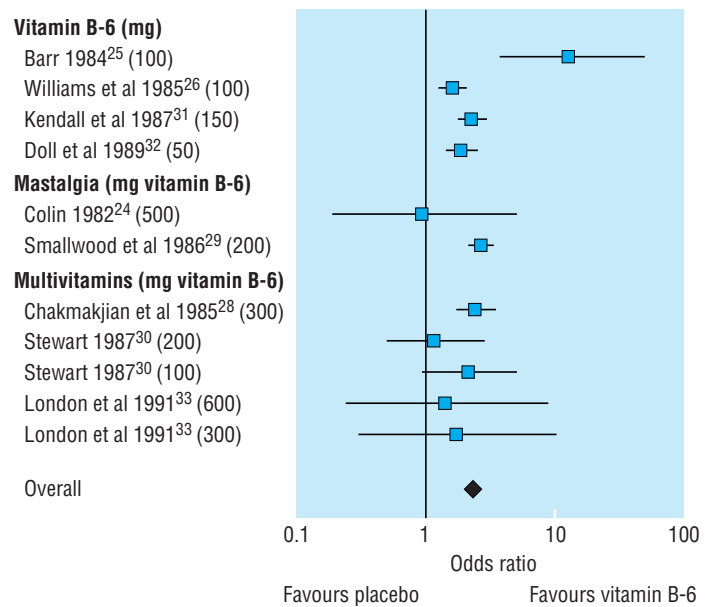

Fig 1 Odds ratios (95\% confidence intervals) for proportion of patients showing improvement in overall premenstrual symptoms with vitamin B-6

\section{Trials}

Vitamin B-6 (depressive symptoms) Williams et al $1985^{26}$ (depression) Kendall et al $1987^{31}$ (negative affect) Doll et al $1989^{32}$ (emotional)

\section{Multivitamins}

(mg vitamin B-6; symptom subgroup) Chakmakjian et al $1985^{28}$ (PMT-D) London et al $1991^{33}$ (600; PMT-D) London et al $1991^{33}$ (300; PMT-D)

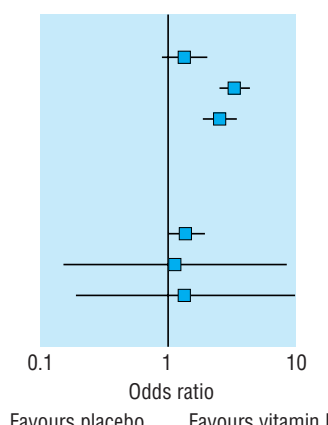

Favours placebo Favours vitamin B- 6

Fig 2 Odds ratios (95\% confidence intervals) for proportion of patients showing improvement in depressive premenstrual symptoms with vitamin B-6

include the trials of mastalgia, as breast pain and breast swelling are frequent premenstrual symptoms. As many as $60 \%$ of women with premenstrual syndrome report cyclical breast pain as a primary component. ${ }^{46}$

The odds ratios in the four included trials that used only vitamin B-6 and studied premenstrual symptomatology 263132 were found to be heterogeneous. The combined odds ratio for the four trials was 2.15 (95\% confidence interval 1.79 to 2.59 ; homogeneity test, $\mathrm{P}=0.035$ ), and when one heterogeneous trial was excluded, ${ }^{25}$ the recalculated odds ratio was 2.07 (1.72 to 2.50; homogeneity test, $\mathrm{P}=0.61$ ).

The use of funnel plots in meta-analysis gives a simple graphical method of assessing bias. A regression analysis to assess quantitatively potential asymmetry of the funnel plot shown in figure 4 indicated no significant asymmetry. ${ }^{22}$ The size and quality of the included trials, however, makes any definite statement concerning bias inappropriate.

\section{Depression}

One of the rationales behind vitamin B-6 being recommended as a treatment for premenstrual syndrome was the observation that it could ease induced depression in several conditions ${ }^{47-49}$ particularly that associated with contraceptive pills high in oestrogen and progesterone. ${ }^{50}{ }^{51}$ The combined odds ratio of the four trials (representing 541 patients) that presented data for depressive symptoms was 1.69 (1.39 to 2.06), and this was homogeneous. This indicates that vitamin B-6 is better than placebo in treating premenstrual depression. It is unlikely, however, that this is the central mode of action of vitamin B-6 in treating premenstrual syndrome, as the odds ratio for overall symptomatology is more favourable than when considering premenstrual depression alone.

\section{Dose response}

The lack of a dose response (fig 3) indicates that the amount of vitamin B-6 given has no impact on the efficacy of its use as treatment for premenstrual syndrome, for either vitamin B-6 alone or when given in a multivitamin preparation. This result adds to the misgivings surrounding the use of vitamin B- 6 as a treatment. The toxic effects of vitamin B-6 at doses higher than 200 $\mathrm{mg} /$ day are well characterised. As the recommended daily allowance of vitamin B-6 is around $2 \mathrm{mg},{ }^{8}$ any

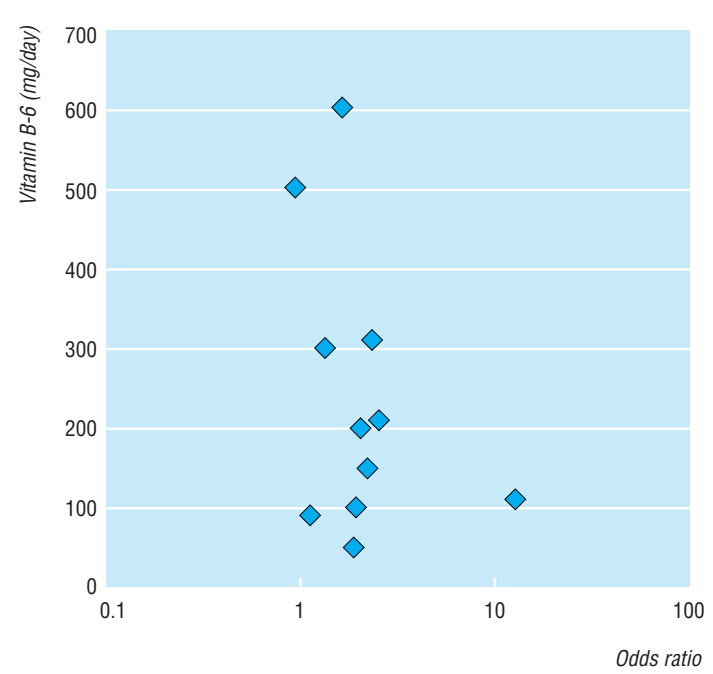

Fig 3 Dose response of vitamin B-6 against individual odds ratios for patients who showed improvement in premenstrual symptoms with vitamin B-6 (when two trials used same dose, data points offset for clarity)

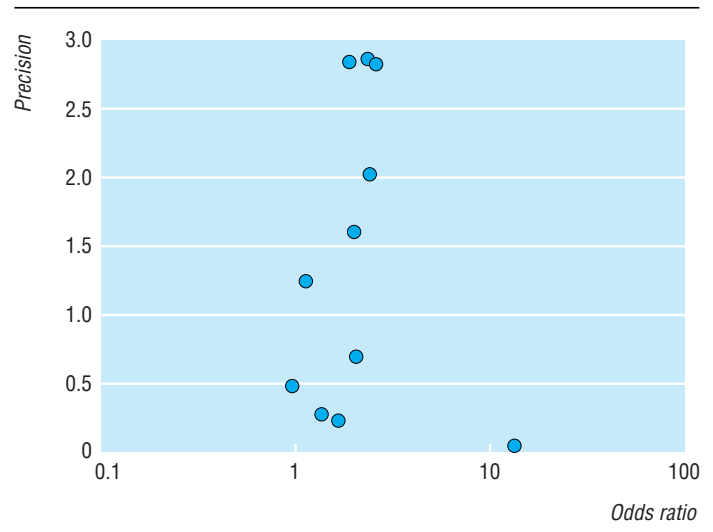

Fig 4 Funnel plot of included studies: precision of study (inverse of $\mathrm{SE})$ against odds ratios 
effect of vitamin B-6 on premenstrual symptomatology would be pharmacological. However, there does remain some controversy as to the precise vitamin B-6 requirement in disease states, highlighted by the successful use of vitamin B-6 doses of around 100 $\mathrm{mg} /$ day in the management of carpal tunnel syndrome. $^{52}$

\section{Multivitamins}

The rationale for including multivitamin treatments in a review on vitamin B-6 is the very large amount of vitamin B-6 in the tablets used, $50 \mathrm{mg}$ per tablet $(600$ $\mathrm{mg}$ /day), which represents over 25 times the recommended daily allowance. ${ }^{8}$ Figure 1 shows the results of the published trials on use of multivitamins in the treatment of premenstrual syndrome, with an overall odds ratio of 2.08 (1.55 to 2.78; homogeneity test, $\mathrm{P}=0.61$ ). The lack of a dose response makes the daily consumption of more than $100 \mathrm{mg}$ of vitamin B-6 inadvisable, and doses in excess of $200 \mathrm{mg}$ /day cannot be recommended in the light of the proved toxic side effects of vitamin B-6, even in a multivitamin preparation.

\section{Side effects}

Only one patient of the 940 participating in these trials indicated the presence of any side effects that could be attributed to the neuropathy associated with pyridoxine toxicity. ${ }^{33}$ This may be due to the comparatively low doses used and the short duration of the trials. The symptoms, however, may have been missed owing to the lack of assessment by a suitably qualified neurologist. Detailed animal studies on pyridoxine indicate that nerve damage can occur before manifestation of the gross symptoms such as ataxia and neuropathy. ${ }^{11}{ }^{12}$

\section{Conclusions}

Conclusions from this meta-analysis are limited by the quality of the trials. No conclusive evidence of vitamin B-6 toxicity was reported, and there seems to be no dose related response to treatment. We conclude, therefore, that there is no rationale for giving daily doses of vitamin B-6 in excess of $100 \mathrm{mg}$. Such doses, and possibly doses of $50 \mathrm{mg}$ /day, are likely to be of benefit in relieving premenstrual symptoms.

We believe that this systematic review does highlight the need for a randomised placebo controlled trial, which should be conducted with sufficient subjects and should have the power to detect any significant clinical difference between vitamin B-6 and placebo.

We thank Mrs Cornelia Dean for translating reference 42, and Mrs Olive Ford (National Association for Premenstrual Syndrome) and Ms Ruth Jepson (Cochrane Menstrual Disorders and Subfertility Group) for assistance with literature surveys.

Contributors: PMSO'B originated the idea for the study and coauthored the paper. He also provided the clinical input to the text and resolved any areas of disagreement in the scoring and data extraction from the trials. KMW was the lead author of the paper and extracted and assessed the trial data. She also assisted in the literature searches, reference collation, data entry, and statistical analysis. PWD undertook the literature searches and located the references and assisted KMW in the data extraction and scoring of the trials. He coordinated the data analysis and statistical calculations in collaboration with KMW and under the guidance of the statistical adviser, PWJ. PWJ gave statistical input
Key messages

- Randomised placebo controlled studies of vitamin B-6 treatment for premenstrual symptoms were of insufficient quality to draw definitive conclusions

- Limited evidence exists to suggest that $100 \mathrm{mg}$ of vitamin B-6 daily (and possibly $50 \mathrm{mg}$ ) are likely to be beneficial in the management of premenstrual syndrome

- Vitamin B-6 was significantly better than placebo in relieving overall premenstrual symptoms and in relieving depression associated with premenstrual syndrome, but the response was not dose dependent

- No conclusive evidence was found of neurological side effects with these doses

- A randomised controlled trial of sufficient power and quality is needed to compare vitamin B-6 with placebo to establish definitive recommendations for treatment

on the data extraction, validity of statistical tests, conversion of statistical measures, and assessment of the heterogeneity of the collated data. He also performed the power calculations and heterogeneity tests and advised on the statistical methods used in the meta-analysis. PWD and KMW will act as guarantors for the paper.

Funding: None.

Competing interests: PMSO'B has been reimbursed for lectures and conferences by the following companies: Shire Pharmaceuticals, SmithKline Beecham, Eli Lilly, Searle, Sanofi Winthrop, Zeneca, and Solvay Pharmaceuticals. He has also received funds for research staff from Searle, SmithKline Beecham, Eli Lilly, and Sanofi Winthrop. He is married to a member of the research department of Zeneca Pharmaceuticals. KMW, PWD, and PWJ declare no competing interests.

1 Joint Food Safety and Standards Group. Survey of dietary supplements containing vitamin B6. London: Ministry of Agriculture Fisheries and Food, 1997.

2 O'Brien PMS. Premenstrual syndrome. London: Blackwell Scientific, 1987.

O'Brien PMS. Helping women with premenstrual syndrome. BMJ 1993;307:1471-5.

4 Smith R, Studd J. Premenstrual syndrome. Clin Rev Gynaecol 1994;939-47.

5 Frank RT. The hormonal causes of premenstrual tension. Arch Neurol Psychiatry 1931;26:1053.

6 Chakmakjian ZH. A critical assessment of therapy for the premenstrual tension syndrome. J Reprod Med 1983;28:532-8.

7 O'Brien PMS. The premenstrual syndrome: a review of the present status of therapy. Drugs 1982;24:140-51.

8 Driskell JA. Vitamin B6 requirements of humans. Nutr Res 1994;14:293324.

9 Abraham GE. Nutritional factors in the etiology of the premenstrual tension syndrome. J Reprod Med 1983;28:446.

10 Antopol W, Thomas M. Experimental study of the effects produced by large doses of vitamin B6.J Neuropathol Exp Neurol 1942;1:330-6.

11 Schaeppi U, Krinke G. Pyridoxine neuropathy: correlation of functional tests and neuropathology in beagle dogs treated with large doses of vitamin B6. Agents Actions 1982;12:575-82.

12 Krinke G, Naylor DC, Skorpil V. Pyridoxine megavitaminosis: an analysis of the early changes induced with massive doses of vitamin $\mathrm{B} 6$ in rat primary sensory neurons. J Neuropathol Exp Neurol 1985;44:117-29.

13 Windebank AJ. Neurotoxicity of pyridoxine analogs is related to coenzyme structure. Neurochem Pathol 1985;3:159-67.

14 Parry GJ. Sensory neuropathy with low dose pyridoxine. Neurology 1985;35:1466-8.

15 Windebank AJ, Low PA, Blexrud MD, Schmelzer JD, Schaumburg HH. Pyridoxine neuropathy in rats: specific degeneration of sensory axons. Neurology 1985;35:1617-22.

16 Berger AR, Schaumburg HH, Schroeder C, Apfel S, Reynolds R. Dose response, coasting, and differential fiber vulnerability in human toxic neuropathy: a prospective study of pyridoxine neurotoxicity. Neurology 1992;42:1367-70.

17 Schaumburg HH, Kaplan J, Windebank AJ, Vick N, Rasmus S, Pleasure D, et al. Sensory neuropathy from pyridoxine abuse: a new megavitamin syndrome. N Eng J Med 1983;309:445-8. 
18 Malmgren R, Collins A, Nilsson CG. Platelet serotonin uptake and effects of vitamin B6-treatment in premenstrual tension. Neuropsychobiology 1987;18:83-6.

19 Jadad A, Moore M, Carrol D, Jenkinson C, Reynolds DJM, Gavaghan DJ, et al. Assessing the quality of reports of randomised clinical trials; is blinding necessary? Control Clin Trials 1996;17:1-12

20 Computer programs for epidemiologic analysis: PEPI version 2. Gahlinger PM and Abramson JH. 2. Stone Mountain, GA: USD, 1995.

21 Hasselblad V, Hedges LV. Meta-analysis of screening and diagnostic tests. Psychol Bull 1995;117:167-78.

22 Egger M, Smith GD, Schneider M, Minder C. Bias in meta-analysis detected by a simple, graphical test. $B M J$ 1997;315:629-34.

23 Stokes J, Mendels J. Pyridoxine and premenstrual tension. Lancet 1972;i:1177-8.

24 Colin C. Studies on the treatment of mastalgia. Rev Med Brux 1982;3:605-9.

25 Barr W. Pyridoxine supplements in the premenstrual syndrome. Practitioner 1984;228:425-7.

26 Williams MJ, Harris RI, Dean BC. Controlled trial of pyridoxine in the premenstrual syndrome. J Int Med Res 1985;13:174-9.

27 Hagen I, Nesheim BI, Tuntland T. No effect of vitamin B-6 against premenstrual tension. A controlled clinical study. Acta Obstet Gynecol Scand 1985;64:667-70.

28 Chakmakjian ZH, Higgins CE, Abraham GE. The effect of a nutritional supplement, Optivite for women, on premenstrual tension syndrome J Appl Nutr 1985;37:12-7.

29 Smallwood J, Ah-Kye D, Taylor I. Vitamin B6 in the treatment of pre-menstrual mastalgia. Br J Clin Pract 1986;40:532-3.

30 Stewart A. Clinical and biochemical effects of nutritional supplementation on the premenstrual syndrome. J Reprod Med 1987;32:435-41.

31 Kendall KE, Schnurr PP. The effects of vitamin B6 supplementation on premenstrual symptoms. Obstet Gynecol 1987;70:145-9.

32 Doll H, Brown S, Thurston A, Vessey M. Pyridoxine (vitamin B6) and the premenstrual syndrome: a randomized crossover trial. J R Coll Gen Pract 1989;39:364-8.

33 London RS, Bradley L, Chiamori NY. Effect of a nutritional supplement on premenstrual symptomatology in women with premenstrual syndrome: a double blind longitudinal study. I Am Coll Nutr 1991:10:494-9.

34 Kerr GD. The management of the premenstrual syndrome. Curr Med Res Opin 1977;4:29-34

35 Day JB. Clinical trials in the premenstrual syndrome. Curr Med Res Opin $1979 ; 6: 40-5$

36 Abraham GE, Hargrove JT. Effect of vitamin B6 on premenstrual symptomology in women with premenstrual tension syndromes: a double blind crossover study. Infertility 1980;3:155-65.
37 Mattes JA, Martin D. Pyridoxine in premenstrual depression. Hum Nut Appl Nutr 1982;36:131-3.

38 Goei GS, Abraham GE. Effect of a nutritional supplement, Optivite, on symptoms of premenstrual tension. J Reprod Med 1983;28:527-31.

39 Fuchs N, Hakim M, Abraham GE. The effect of a nutritional supplement, Optivite for women, on premenstrual tension syndromes. J Appl Nutr 1985;37:1-11.

40 Brush MG, Bennett T, Hansen K. Pyridoxine in the treatment of premenstrual syndrome: a retrospective survey in 630 patients. Br J Clin Pract 1988;42:448-52.

41 Brush MG. Vitamin B6 treatment of premenstrual syndrome. In: Leklem JE, Reynolds RD, eds. Clinical and physiological applications of vitamin B6. New York: Liss, 1988: 363-79. (Current topics in nutrition and disease, vol 19).

42 Van den Berg H, Schrijver J, Bruinse HW, van der Ploeg HM. Vitamin B6 and premenstrual syndrome. Voeding 1989;50:58-62.

43 Berman MK, Taylor ML, Freeman E. Vitamin B-6 in premenstrual syndrome. J Am Diet Assoc 1990;90:859-61.

44 De Souza MC, Walker AF, Bolland KM, Robinson PA. A synergistic effect of magnesium and vitamin B6 supplementation for the relief of symptoms of the premenstrual syndrome (PMS). Proc Nutr Soc 1996:56:75 A

45 Harrison W, Endicott J, Rabkin JG, Nee J. Treatment of dysphoric changes: clinical outcome and methodological implications. Psychol Bull 1984;118-22.

46 Blue J, Harman J. Mastalgia review: St Marks Breast Centre. NZ Med J 1998;111:34-7.

47 Villegas-Salas E, Ponce dL, Juarez-Perez MA, Grubb GS. Effect of vitamin B6 on the side effects of a low-dose combined oral contraceptive. Contraception 1997;55:245-8.

48 Bernstein AL. Vitamin B6 in clinical neurology. Ann NY Acad Sci 1990;585:250-60.

49 Stewart JW, Harrison W, Quitkin F, Baker H. Low B6 levels in depressed outpatients. Biol Psychiatry 1984;19:613-6.

50 Adams PW, Rose DP, Folkard J, Wynn V, Seed M, Strong R. Effects of pyridoxine hydrochloride upon depression associated with oral contraceptives. Lancet 1973;:897-904.

51 Winston F. Oral contraceptives, pyridoxine, and depression. Am J Psychia try 1973;130:1217-21.

52 Folkers K, Ellis J. Successful therapy with vitamin B6 and vitamin B2 of the carpal tunnel syndrome and need for determination of RDAs for vitamins B6 and B2 for disease states. Ann NY Acad Sci 1989;295-301.

(Accepted 11 March 1999)

\title{
Influence of hospital and clinician workload on survival from colorectal cancer: cohort study
}

\author{
F Kee, R H Wilson, C Harper, C C Patterson, K McCallion, R F Houston, R J Moorehead, J M Sloan,
} B J Rowlands

\begin{abstract}
Objective To determine whether clinician or hospital caseload affects mortality from colorectal cancer. Design Cohort study of cases ascertained between 1990 and 1994 by a region-wide colorectal cancer register.

Outcome measures Mortality within a median follow up period of 54 months after diagnosis.

Results Of the 3217 new patients registered over the period, 1512 (48\%) died before 31 December 1996 Strong predictors of survival both in a logistic regression (fixed follow up) and in a Cox's proportional hazards model (variable follow up) were Duke's stage, the degree of tumour differentiation, whether the liver was deemed clear of cancer by the surgeon at operation, and the type of intervention (elective or emergency and curative or palliative intent). In a multilevel model, surgeon's caseload had no significant effect on mortality at 2 years. Hospital workload, however, had a significant impact on
\end{abstract}

survival. The odds ratio for death within 2 years for cases managed in a hospital with a caseload of between 33 and 46 cases per year, 47 and 54 cases per year, and $\geqslant 55$ cases per year (compared to one with $\leq 23$ cases per year) were respectively $1.48(95 \%$ confidence interval 1.03 to 2.13 ), 1.52 (1.08 to 2.13), and 1.18 (0.83 to 1.68 ).

Conclusions There was no detectable caseload effect for surgeons managing colorectal cancer, but survival of patients treated in hospitals with caseloads above 33 cases per year was slightly worse than for those treated in hospitals with fewer caseloads. Imprecise measurement of clinician specific "events rates" and the lack of routinely collected case mix data present major challenges for clinical audit and governance in the years ahead.

\section{Introduction}

In 1995, the then chief medical officer, Sir Kenneth Calman, made recommendations for improving
Department of Epidemiology and Public Health, Queen's University of Belfast, Belfast BT12 6BJ

F Kee,

professor

C C Patterson, senior lecturer in medical statistic

Northern Ireland Centre for Clinical Oncology, Belvoir Park Hospital, Belfast BT8 8JR R H Wilson, specialist registrar R F Houston, consultant

continued over

BMJ 1999;318:1381-6 